\title{
Comprehensive transcriptional profiling of porcine liver aging
}

\author{
Jianning Chen ${ }^{\text {Equal first author, } 1}{ }^{1}$, Qin Zou ${ }^{\text {Equal first author, } 1 \text {, Daojun } \text { Lv }^{2} \text {, Muhammad Ali Raza }}{ }^{3}$, Xue Wang ${ }^{1}$, Yan Chen ${ }^{1}$, Xiaoyu \\ $\mathrm{Xi}^{1}$, Peilin Li ${ }^{2}$, Anxiang Wen ${ }^{1}$, Li Zhu ${ }^{4}$, Guoqing Tang ${ }^{4}$, Mingzhou $\mathrm{Li}^{4}{ }^{4}$, Xuewei $\mathrm{Li}^{4}{ }^{4}$, Yanzhi Jiang ${ }^{\text {Corresp. } 1}$ \\ 1 \\ ${ }^{1}$ Department of Zoology, Sichuan Agricultural University, Ya'an, Sichuan, China \\ Sichuan Weimu Modern Agricultural Science and Technology Co., Ltd, Chengdu, Sichuan, China \\ 3 Department of Crop Cultivation and Farming System, Sichuan Agricultural University, Chengdu, Sichuan, China \\ ${ }^{4}$ Farm Animal Genetic Resources Exploration and Innovation Key Laboratory of Sichuan Province, Sichuan Agricultural University, Chengdu, Sichuan, \\ China \\ Corresponding Author: Yanzhi Jiang \\ Email address: jiangyz04@163.com
}

Background. Aging is a major risk factor for the development of many diseases, and the liver, as the most important metabolic organ, is significantly affected by aging. It has been shown that the liver weight tends to increase in rodents and decrease in humans with age. Pigs have a genomic structure, physiological as well as biochemical features that are similar to those of humans, and have therefore been used as a valuable model for studying human diseases. The molecular mechanisms of the liver aging of large mammals on a comprehensive transcriptional level remain poorly understood. The pig is an ideal model animal to clearly and fully understand the molecular mechanism underlying human liver aging.

Methods. In this study, four healthy female Yana pigs (an indigenous Chinese breed) were investigated: two young sows (180-days-old) and two old sows (8-years-old). High throughput RNA sequencing was performed to evaluate the expression profiles of messenger RNA, long non-coding RNAs, micro RNAs, and circular RNAs during the porcine liver aging process. Gene Ontology (GO) analysis was performed to investigate the biological functions of age-related genes.

Results. A number of age-related genes were identified in the porcine liver. GO annotation showed that up-regulated genes were mainly related to immune response, while the down-regulated genes were mainly related to metabolism. Moreover, several IncRNAs and their target genes were also found to be differentially expressed during liver aging. In addition, the multi-group cooperative control relationships and constructed circRNA-miRNA co-expression networks were assessed during liver aging. Conclusions. Numerous age-related genes were identified and circRNA-miRNA co-expression networks that are active during porcine liver aging were constructed. These findings contribute to the understanding of the transcriptional foundations of liver aging and also provide further references that clarify human liver aging at the molecular level. 


\section{Comprehensive transcriptional profiling of porcine liver aging}

2 Jianning Chen ${ }^{1, \dagger}$, Qin Zou ${ }^{1, \dagger}$, Daojun $\mathrm{Lv}^{2}$, Muhammad Ali Raza ${ }^{3}$, Xue Wang${ }^{1}$, Yan Chen ${ }^{1}$, Xiaoyu Xi', Peilin

$3 \quad \mathrm{Li}^{2}$, Anxiang Wen${ }^{1}$, Li Zhu ${ }^{4}$, Guoqing Tang ${ }^{4}$, Mingzhou $\mathrm{Li}^{4}$, Xuewei $\mathrm{Li}^{4}$ and Yanzhi Jiang ${ }^{1, *}$

$4{ }^{1}$ Department of Zoology, College of Life Science, Sichuan Agricultural University, Ya'an, Sichuan 625014, China;

$5 \quad{ }^{2}$ Sichuan Weimu Modern Agricultural Science and Technology Co., Ltd., Chengdu Sichuan 611130, China;

6 32Department of Crop Cultivation and Farming System, College of Agronomy, Sichuan Agricultural University,

7 Chengdu, Sichuan 611130, China;

$8{ }^{4}$ Farm Animal Genetic Resources Exploration and Innovation Key Laboratory of Sichuan Province, Sichuan 9 Agricultural University, Chengdu, Sichuan 611130, China.

$10 \dagger$ These authors contributed equally to this work.

J.N. Chen \& Q. Zou

* Correspondence to: Y.Z. Jiang, Department of Zoology, College of Life Science, Sichuan Agricultural University, No. 46, Xinkang Road, Ya'an City 625014, Sichuan Province, China.

Tel.: +86 835 2886103; Fax: +86 8352886080 ;

E-mail addresses: jiangyz04@163.com (Y.Z. Jiang)

\section{Abstract:}

Background. Aging is a major risk factor for the development of many diseases, and the liver, as the most important metabolic organ, is significantly affected by aging. It has been shown that the liver weight tends to increase in rodents and decrease in humans with age. Pigs have a genomic structure, physiological as well as biochemical features that are similar to those of humans, and have therefore been used as a valuable model for studying human diseases. The molecular mechanisms of the liver aging of large mammals on a comprehensive transcriptional level remain poorly understood. The pig is an ideal model animal to clearly and fully understand the molecular mechanism underlying human liver aging. 
Methods. In this study, four healthy female Yana pigs (an indigenous Chinese breed) were investigated: two young sows (180-days-old) and two old sows (8-years-old). High throughput RNA sequencing was performed to evaluate the expression profiles of messenger RNA, long non-coding RNAs, micro RNAs, and circular RNAs during the porcine liver aging process. Gene Ontology (GO) analysis was performed to investigate the biological functions of age-related genes.

Results. A number of age-related genes were identified in the porcine liver. GO annotation showed that up-regulated genes were mainly related to immune response, while the downregulated genes were mainly related to metabolism. Moreover, several lncRNAs and their target genes were also found to be differentially expressed during liver aging. In addition, the multigroup cooperative control relationships and constructed circRNA-miRNA co-expression networks were assessed during liver aging.

Conclusions. Numerous age-related genes were identified and circRNA-miRNA coexpression networks that are active during porcine liver aging were constructed. These findings contribute to the understanding of the transcriptional foundations of liver aging and also provide further references that clarify human liver aging at the molecular level.

\section{Introduction}

The aging process leads to the gradual loss of the ability of an organism to maintain homeostasis and its structure alters. The organism therefore becomes more vulnerable to external stresses or damage (López-Otín et al., 2013). Aging significantly affects a number of physiological and biochemical functions that are performed by the liver (Kitani, 1994). Aging has been suggested as a major risk factor for the development of various diseases (Rodwell et al., 
49

50

51

52

53

54

2002), and several studies showed that aging increased the risks for numerous liver diseases that cause an increase in the mortality rate (Amarapurkar et al., 2007; Regev \& Schiff, 2001;

Sheedfar et al., 2013). Previous studies have shown that the liver size progressively decreased in response to aging and a marked decline in volume was found to occur above the age of 60 years in humans (Woodhouse \& James, 1990). This has been suggested to be related to an agedependent decrease in hepatic blood flow (Wynne et al., 2010; Zoli et al., 1999). The aging liver in humans has been characterized to have macrohepatocytes, polyploidy, and increased numbers of in nuclei and nucleoli (Schmucker, 1998). It has been reported that the rate of hepatic polyploidization accelerated after five decades of life in humans (Kudryavtsev et al., 1993). In the liver, the neural fat and cholesterol volumes gradually expand in response to ageing, and this accumulation of lipids may damage the normal liver function by promoting organ-specific toxic reactions (Kim et al., 2015; Slawik \& Vidal-Puig, 2006). More importantly, the aging process in the liver can lead to a decreased number and dysfunction of mitochondria, as well as a decrease in the area of the smooth endoplasmic reticulum. These effects decrease the generation of smooth endoplasmic reticulum and reduce the synthesis of microsomal proteins (Kim et al., 2015). The changes of structure and dysfunction in the liver in response to age were considered to be associated with a significant impairment of many hepatic metabolic and detoxification activities (Bertolotti et al., 2014).

Due to the continuous development of molecular biology, the aging process is currently understood not only a process accompanied by morphological changes, but also as a process that involves complicated changes at the molecular level. Recently, several studies have shown that DNA methylation, as an important part of epigenetics, changes during human liver aging (Bacalini et al., 2018; Bysani et al., 2016; Huse et al., 2015). A number of DNA methylation 
72 changes have been found to be associated with gene expression during aging in the liver (Bysani

73 et al., 2016). Moreover, numerous gene expression changes have been reported during aging in

74 the mouse liver (Kwekel et al., 2010; White et al., 2015), and age-related genes have been

75 reported to be involved in immune response, metabolic processes, cell activation, and RNA modification (White et al., 2015). In addition, the non-coding RNAs including microRNAs (miRNAs), long non-coding RNAs (lncRNAs), and circular RNAs (circRNAs) have been found to play an important role in the regulation of gene expression (Chen \& Yang, 2015; Eulalio et al., 2008; Mercer et al., 2009), and to be closely related to many diseases (Chen et al., 2013; Feng et al., 2014; Lukiw, 2013; Zhao et al., 2016). Furthermore, numerous miRNAs and lncRNAs were identified to be differentially expressed during liver aging in both humans and mice (Capri et al., 2017; Shima et al., 2014; White et al., 2015). However, the changes involved in the comprehensive transcriptome, including mRNA, lncRNA, miRNA, and circRNA, during liver aging remain largely unknown both in humans and other big mammals. More importantly, previous studies have shown that the liver weight tends to increase in rodents, while it decreases in humans with age (Kitani, 1994). Therefore, the mouse may not be a good model animal to clearly and fully understand the underlying molecular mechanisms of human liver aging. It has been reported that pigs have a genomic structure, as well as both physiological and biochemical features that are similar to those of humans. The prominent exception is their anatomic structure (Welsh et al., 2009), which is more similar than that of mice (Wernersson et al., 2005). The pig has been identified as a valuable model to study human diseases (Lunney, 2007; Zhu et al., 2015). The above-mentioned information suggests that the pig may be an ideal model animal to obtain an understanding of the aging of the human liver at the transcriptional level.

94 Puberty and reproductive exhaustion are the two key points in the reproductive development 
95 of pigs, and the median ages of both sow puberty and reproductive exhaustion are about 180 96 days and 8 years, respectively (Jones et al., 1998). Both of these critical time points correspond

97 to the age of 14 and 50 in humans, respectively (Hansen et al., 2008). Moreover, the liver has

98 been found to be in a recession at the age of 50 in humans, and the aging rate entered a period of accelerated decline after the age of 50 (Kudryavtsev et al., 1993). To better understand the comprehensive transcriptional profiling of liver aging, pigs were used as model animals and an integrated multi transcriptome-wide profiling (mRNA, miRNA, lncRNA, and circRNA) analysis was performed via high-throughput sequencing of the liver from two young (180 days) and two old (8 years) sows. This procedure enabled to identify numerous age-related genes and unclear multi-group cooperative control networks in the aging pig liver. In summary, the obtained data may help to explain age-associated changes in the transcriptional patterns during liver aging, and also provide further references toward an understanding of human liver aging at the molecular level.

\section{Materials \& Methods}

\section{Animals}

In this experiment, four Yanan sows were used to investigate the porcine liver aging process. For this study, two young sows (180-days-old) and two old sows (8-years-old) were selected. Moreover, no direct and collateral blood relationship existed among the four pigs within the last three generations of these experimental animals. All experiments involving animals were conducted according to the Regulations for the Administration of Affairs Concerning Experimental Animals (Ministry of Science and Technology, China, revised in June 2004), and approved by the Institutional Animal Care and Use Committee in College of Animal Science and Technology, Sichuan Agricultural University (Sichuan, China) under permit No. SKY- 
B20160201.

Feeding and slaughtering standards of the pigs were performed as described in previous literature (Chen et al., 2018). As in previous research, piglet weaning was executed at the age of $28 \pm 1$ days. After weaning, an initial diet consisting of $3.40 \mathrm{Mcal} \mathrm{kg}^{-1}$ metabolizable energy having $20.0 \%$ crude protein $\left(11.5 \mathrm{~g} \mathrm{~kg}^{-1}\right.$ lysine $)$ was provided to piglets, which continued from day 30 to 60 . The pigs were shifted to a different diet, composed of $14.0 \mathrm{MJ} \mathrm{kg}^{-1}$ of metabolizable energy and $18.0 \%$ crude protein $\left(9.0 \mathrm{~g} \mathrm{~kg}^{-1}\right.$ lysine $)$ from the $61^{\text {st }}$ to the $120^{\text {th }}$ day. On the $121^{\text {st }}$ day, pigs were subjected to a feed constituting $16.0 \%$ crude protein $\left(8.0 \mathrm{~g} \mathrm{~kg}^{-1}\right.$ lysine) and $13.5 \mathrm{MJ} \mathrm{kg}^{-1}$ of metabolizable energy. Similar conditions were provided to pigs with the permission to freely access food and water. Pigs were not allowed to access food one night before slaughtering and had a resting period of $2 \mathrm{~h}$ after transportation. To exsanguinate and relief the pain, a sudden electric shock of $90 \mathrm{~V}$ and $50 \mathrm{~Hz}$ for $10 \mathrm{~s}$ was administered (Chen et al., 2018).

\section{Sample preparation}

As previous study described, all samples were investigated following the rules and regulations for the handling and establishment of research animals by the Ministry of Science and Technology of China (Chen et al., 2018). Porcine liver tissue was harvested from each pig and frozen in liquid nitrogen. After that, samples were kept in a freezer at $-80{ }^{\circ} \mathrm{C}$ until RNA extraction. The TRIzol Reagent (Invitrogen, CA, USA) was used to extract the total RNA, which was subsequently treated with DNase and purified by using the RNeasy Mini Kit (Qiagen, CA, USA). The quality and concentration of RNA were determined by an Agilent Bioanalyzer 2100 system.

\section{RNA sequencing}

RNA samples were prepared by using about $5 \mu \mathrm{g}$ RNA per sample. According to the 
143

144

145

146

147

148

149

150

151

152

153

154

155

156

157

158

159

160

161

162

163

164

165

166

manufacturer's information, the rRNA-depleted RNA by NEBNext ${ }^{\circledR}$ Ultra ${ }^{\mathrm{TM}}$ Directional RNA

Library Prep Kit for Illumina ${ }^{\circledR}$ (New England Biolabs Ipswich, Massachusetts, USA) was used to obtain sequencing libraries. The Agilent Bioanalyzer 2100 system was utilized to analyze the library quality. After clusters had been generated, RNA sequencing was performed using Illumina HiSeq 4000 and 150 bp paired-end reads were obtained.

\section{Small RNA sequencing}

A small RNA library was prepared by taking a total of $5 \mu \mathrm{g}$ of RNA per sample as input material. To generate sequencing libraries, NEBNext ${ }^{\circledR}$ Multiplex Small RNA Library Prep Set for Illumina ${ }^{\circledR}$ (New England Biolabs Ipswich, Massachusetts, USA) was used, according to the manufacturer's information. To attribute sequences to each sample, index codes were supplemented. Here, the Agilent Bioanalyzer 2100 system was also utilized to test the quality of the library. According to the information provided by the manufacturer, a cBot Cluster Generation System using TruSeq SR Cluster Kit v3-cBot-HS (Illumina, Inc, California, USA) was used to cluster the index-coded samples. After the clusters had been generated, the Illumina MiSeq platform was used to sequence the obtained library preparations, and $50 \mathrm{bp}$ single-end reads were obtained.

\section{Statistical analysis}

Clean reads of RNA sequencing were obtained from raw data after eliminating the adapter, poly-N, and low-quality reads. The clean reads were aligned to the reference genome database (Ensemble Susscrofa 10.2) using TopHat2 (v2.0.14) and default parameters were used (Kim et al., 2013). Mapped reads per sample were assembled by using the StringTie software (Pertea et al., 2015), which at least presented in one of the two replicates. Mapped transcripts blasted (evalue $=1 \mathrm{e}-10)$ to Ensemble were directly described as known mRNA or lncRNA. To estimate the transcripts per million (TPMs) of both lncRNAs and mRNA, Salmon (v0.6.0) was utilized 
167 (Patro et al., 2015). To examine the transcript coding potential, Coding Potential Calculator 168 (CPC) (0.9) (Kong et al., 2007) and Pfam Scan (v1.5) (Punta et al., 2012) were utilized. Coding 169 potential transcripts predicted by one or both tools were filtered out, and non-coding potential 170 transcripts were identified as the candidate set of novel lncRNAs. The circRNAs were predicted

171

by CIRCexplorer2 (junction reads $\geq 2$ ) (Zhang et al., 2016).

The miRBase21 was used as reference and the software mirdeep2 (Friedländer et al., 2011) was utilized to identify the known miRNA and to predict novel miRNA. The miRanda (v3.3a) software (Betel et al., 2008) was used to predict the target genes of miRNAs and default parameters and cutoffs (Score $S \geq 140$ and Energy $E \leq-20.0$ ) were used. Later, the expression levels of miRNAs were also measured by TPMs.

The mRNAs, lncRNAs, miRNAs, and circRNAs were identified to be differentially expressed by using the edgeR package (Robinson et al., 2010) in the R programming environment. Moreover, in the "classic analysis" section, the edgeR user guidelines were adopted in detail. A $q$-value $<0.05$ and fold change $>2$ were used to determine differentially expressed mRNAs, lncRNAs, and miRNAs in the old group vs. young group. For differentially expressed circRNAs, the standard $p$-value $<0.05$ and a fold change $>2$ was used. Here, the fold change of mRNAs, lncRNAs, miRNAs, and circRNAs was the $\log 2$ transformed ratio of the average expression between two groups, and was estimated by edgeR.

The GOseq R package was used to perform the Gene Ontology (GO) enrichment analysis, and GO terms with a corrected $p$-value $<0.05$ were considered to be significantly enriched by the genes. Principal Component Analysis (PCA) was performed by the prcomp function in R. All the Pearson's correlation coefficient analysis in our research used the cor function in R programming. 
190

191

192

193

194

195

196

197

198

199

200

201

202

203

204

205

206

207

208

209

210

211

212

213

\section{CircRNA-miRNA co-expression network}

The correlation analysis was the base for the construction of a circRNA-miRNA coexpression network between the differentially expressed circRNA and miRNAs. The Pearson's correlation coefficient $(r)$ was used for the expression analysis of differentially expressed circRNAs and miRNAs. The $r>0.8$ and a $p$-value $<0.05$ were considered relevant for network construction between a circRNA and a miRNA.

\section{Quantitative PCR validation}

The oligo (dT) and random 6-mer primers given in the PrimeScript RT Master Mix kit (TaKaRa, Shiga, Japan) were utilized for cDNA synthesis. The CFX96 Real-Time PCR detection system (Bio-Rad Laboratories, Inc., Hercules, California, USA) was used to conduct qPCR using the SYBR Premix Ex Taq kit (Takara, Shiga, Japan). Three biological replicates were taken to perform the q-PCR analysis. In this assay, three endogenous control genes (porcine $G A P D H, A C T B$, and $U 6$ snRNA) were used. The expression levels of objective mRNAs, miRNAs, lncRNAs, and circRNAs were determined by the $2^{-\Delta \Delta \mathrm{Ct}}$ method. The Pearson's correlation coefficient of the $\log 2$ fold change values between RNA-seq and q-PCR was used to validate the reliability of the obtained RNA-seq data.

\section{Results}

\section{Transcriptome profile of the aging liver}

To determine the changes in transcriptome level due to aging in the liver, four porcine liver tissues were obtained from two age groups. Here, RNA sequencing (RNA-seq) was used to explore transcriptome-wide profiling including mRNA, miRNA, lncRNA, and circRNA. An estimate $\sim 71.66$ million clean reads were achieved per RNA-seq library. An alignment of $\sim 73.40-79.40 \%$ of clean reads were found with the porcine reference genome (Ensemble Susscrofa 10.2) (Table S1). Moreover, 8.00-9.43 million clean reads were gained per small 
214 RNA-seq library. 74.39-80.24\% alignment of clean reads were mapped reads (Table S2). Among

215 these four samples (including two young livers (YL) and two old livers (OL)), a total of 21607

216 mRNAs, 4028 lncRNAs, 888 miRNAs, and 6366 circRNAs were detected (Fig. 1, Dataset S1).

217 Out of 888 miRNAs, 336 miRNAs were identified as novel miRNAs (Fig. 1E). 84.5\%, 64.4\%,

218 and $95.2 \%$ of these identified mRNAs, lncRNAs, and miRNAs, respectively, were expressed in

219 both young and old groups; however, only circRNAs had an expression of $16.9 \%$. Moreover, the

220 number of only expressed mRNAs, lncRNAs, miRNAs, and circRNAs in OL exceeded those

221 only expressed in YL (Fig. 1, Dataset S2).

222 Unsupervised Euclidean matrix plots for expressed mRNA and lncRNA showed that both the

223 old individuals and younger individuals were grouped separately (Fig. 2A), indicating an age-

224 dependent expression pattern. Moreover, a closer distance was found between old individuals

225 compared to that of younger individuals. PCA was used for the identified mRNA and lncRNA

226 transcripts, and the results showed that the young group had a high degree of variance between

227 each biological replicate, while old individuals clustered (Fig. 2B). Furthermore, four samples

228 were investigated using unsupervised Euclidean matrix plots based on miRNAs (Fig. 2C). The

229 result also indicated age-dependent expression patterns. However, the result of PCA for miRNA

230 has shown that there was a higher degree of variance between each biological replicate in the old

231 group than in the young group (Fig. 2D), which contrasted with the PCA result based on mRNA

232 and lncRNA.

233

234

235

236

237

\section{Differentially expressed mRNAs during liver aging}

Form our results, though most genes had been shared for both ages during liver development, we also detected a proportion of age-dependent differentially expressed genes (DEGs). First, DEGs were screened in OL vs. YL (OYL), which identified 273 DEGs, of which 238 were upregulated and 35 were down-regulated (Fig. 3A, Dataset S3). To explore the biological functions 
238 of these age-related genes, GO analysis was performed. Compared to the young group, the

239 results showed that the up-regulated genes were significantly enriched for immune response, the

240 regulation of cell differentiation, stress response, both transport and storage of lipids, such as

241 defense response to virus (GO:0051607), and ISG15-protein conjugation (GO:0032020)。

242 Positive regulation was found for cell differentiation (GO:0045597), response to biotic stimulus

243 (GO:0009607), positive regulation of lipid storage (GO:0010884), and chylomicron remnant

244 clearance (GO:0034382) (Fig. 3C, Dataset S4). For example, the gene nuclear receptor subfamily

2451 group H member 2 (NR1H2, ENSSSCG00000003211) was identified to be up-regulated (log

$246 \mathrm{FC}=2.026481405)$ (Dataset S3) during liver aging, and it was GO enriched for the positive

247 regulation of lipid storage (Dataset S4). The NR1H2 encoding liver X receptor (LXR)- $\beta$ was

248 found to be universally expressed (Kalaany \& Mangelsdorf, 2006), and the variation within the

249 NR1H2 gene may facilitate the development of type 2 diabetes (Ketterer et al., 2011). Moreover,

250 the genetic variability at $\mathrm{NR} 1 \mathrm{H} 2$ may contribute to an increased risk of Alzheimer's disease

251 (Adighibe et al., 2006), and the preeclampsia was associated with a polymorphism in NR1H2

252 (Mouzat et al., 2011).

253 The down-regulated genes were mainly related to diphosphatase activity, signaling pathway,

254 and metabolic processes, such as phosphodiesterase I activity (GO:0004528), nucleotide

255 diphosphatase activity (GO:0004551), regulation of nodal signaling pathway (GO:1900107),

256 ethanol metabolic process (GO:0006067), and lipid metabolic processes (GO:00066295) (Fig.

257 3C, Dataset S4). The ELOVL fatty acid elongase 2 gene (ELOVL2, ENSSSCG00000001045)

258 was found to be closely related to lipid metabolic processes (Dataset S4), and was found to be

259 significantly down-regulated $(\log \mathrm{FC}=-3.306051731)$ (Dataset $\mathrm{S} 3)$. A previous study has

260 reported ELOVL2 as a highly expressed gene in the liver that was closely related to the 
261 elongation process of PUFAs with 22 carbons to produce 24-carbon precursors for DHA and

262 DPAn-6 formation, indicating that it was essential for lipid homeostasis (Pauter et al., 2014). In

263 addition, in testis, the ELOVL2 can also control the level of n-6 28:5 and 30:5 fatty acids, which

264 had been identified as indispensable for normal sperm formation and fertility in male mice

265 (Zadravec et al., 2011).

Differentially expressed noncoding RNA during liver aging

267 To detect the expression profiling of age-related lncRNA, the identified lncRNAs were

268 screened out during porcine liver aging. Here, 19 lncRNAs were identified as differentially expressed lncRNAs (DELs) in the aging of porcine liver. 17 out of these 19 lncRNAs presented up-regulation, and 2 exhibited down-regulation (Fig. 3B, Dataset S3). Additionally, GO analysis was performed to investigate the target genes of DELs bearing biological functions. The results showed that the target genes of up-regulated lncRNAs were significantly enriched for the immune response, signaling pathway, and protein transport, such as positive regulation of type I interferon production (GO:0032481), defense response to virus (GO:0051607), positive regulation of NF-kappaB transcription factor activity (GO:0051092), response to virus (GO:0009615), positive regulation of RIG-I signaling pathway (GO:1900246), epoxygenase P450 pathway (GO:0019373), and SMAD protein import into nuclei (GO:0007184) (Fig. 3D, oxidation-reduction processes, metabolic processes, diphosphatase activity, iron ion and heme binding, and transport processes, such as oxidation-reduction process (GO:0055114), oxidoreductase activity (GO:0016491), metabolic process (GO:0008152), phosphodiesterase I activity (GO:0004528), nucleotide diphosphatase activity (GO:0004551), iron ion binding (GO:0005506), heme binding (GO:0020037), lipid transport (GO:0006869), and both bile acid and bile salt transport (GO:0015721) (Fig. 3D, Dataset S5). 
To investigate the co-regulated relationships of lncRNA-mRNA during liver aging, the DELs

286

287

288

289

290

291

292

293

294

295

296

297

298

299

300

301

302

303

304

305

306

307

and their predicted target genes were screened, and three significantly up-regulated lncRNAs (MSTRG.58269, MSTRG.85782, and MSTRG.177174; $\operatorname{logFC}>9.0$ ) as well as one downregulated lncRNA (MSTRG.205482; $\operatorname{logFC}<-2.0)$ were found. Several of their target genes were differentially expressed during liver aging (Table 1). Here, MSTRG.58269 was found to have biggest change in expression $(\operatorname{logFC}=9.880537444)$, and its target genes $D M B X 1$ (enriched for DNA binding) and GUCA1A (enriched for Calcium sensitive guanylate cyclase activator activity) were both up-regulated in response to liver aging. Furthermore, most of the target genes of MSTRG.85782 were up-regulated during liver aging, and these genes were significantly enriched for immune response, such as IRG6 and ISG15 (enriched for the defense response to virus), SIPR3 (regulation of interleukin-1 beta production), and IRG6 (negative regulation of viral genome replication). In addition, the target genes of MSTRG.205482 were down-regulated in response to liver aging, and were significantly enriched for metabolic process, such as LIPK (related to lipid metabolic process), SULT1B1 (related to flavonoid metabolic process), and TLR4 (related to lipopolysaccharide receptor activity) (Table 1).

A total of 26 differentially expressed miRNAs (DEMs)were identified in OYL, of which 14 were up-regulated and 12 were down-regulated (Fig. S1A, Dataset S3). Moreover, 103 differentially expressed circRNAs (DECs)were identified in OYL, of which 81 were upregulated and 22 were down-regulated (Fig. S1B, Dataset S3). Here, the $m i R-206(\operatorname{logFC}=$ 5.300862103) was identified to be significantly up-regulated in response to liver aging (Dataset S3). A previous study has shown that $m i R-206$ can regulate the glucokinase activity that knocked out the miR-206 and produced the elevated glycogen content in the mice liver (Manjula et al., 2016). Moreover, it was found that $m i R-206$ represses the LXR- $\alpha$ activity and expression in 
308 hepatic cells (Vinod et al., 2014).

309 The circRNA as competitive endogenous RNA (ceRNA) regulate the function of miRNA

310 acting as a 'sponge', thus it could regulate the expression of target genes. This indicates that

311 circRNAs and their target miRNAs may exert a co-expression during liver aging (Li et al., 2015;

312 Sanger et al., 1976). Here, the Pearson correlation coefficien was analyzed between circRNA and

313 miRNA based on the expression levels of DECs and DEMs, and a co-expression network map

314 was constructed (Dou et al., 2016). The network map included 5 miRNAs, 13 circRNAs, and 13

315 relationships (Fig. 4, Dataset S6). In this network, 11 circRNAs associated with three miRNAs

316 were found to be synchronously up-regulated. However, two circRNAs associated with two

317 miRNAs were found to be synchronously down-regulated. In addition, seven circRNAs with

318 GL893173.1_33471 (novel miRNA) presented most relationships, while circRNA003190 and

319 circRNA017214 showed single relationship with $m i R-299$ and $m i R-210$, respectively (Fig. 4,

320 Dataset S6).

The expression of genes validated by q-PCR

q-PCR was performed to verify the RNA-seq results. A certain number of genes were chosen

323

324

325

326

327

328

329

330

331

that were found to play a significant role in specific diseases or in vital biology functions.

Moreover, several DEGs were randomly selected to conduct q-PCR to determine the expression

levels of these gene in the four samples (two 180-days pigs and two 8-years pigs). Selected genes were composed of two mRNAs (ELOVL2 and NR1H2), two lncRNAs (MSTRG.6003 and MSTRG.205482), two miRNAs (miR-210 and miR-299), and two circRNAs (circRNA005502

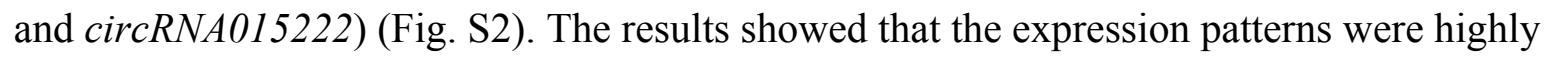
consistent between the two methods $(r=0.887581, p=0.003259)$ (Fig. S2).In this study, the $\mathrm{q}$ PCR results affirmed the high reproducibility and reliability of the gene expression profiling from RNA-seq results. The primer pairs used here are listed in Table S3. 
In this study, we described changes of comprehensive transcriptome profiling in porcine liver during aging and a large number of mRNAs, lncRNAs, and miRNAs were found expressed in both young and old livers. However, $\sim 16.9 \%$ of cirRNAs were found expressed in both young and old livers, which suggests that the expression of circRNA showed a strong stage-specific pattern during aging. This may indicate that the circRNA not only regulates gene expression via competitive binding of miRNAs as ceRNAs, but is also involved in many vital roles during liver aging in mammals. Previous studies showed that circRNAs could translate to proteins and play significant roles in the inhibition of cancer development or biological functions (Yun et al., 2017; Zhang et al., 2018). This study presented that young and old individuals were separately grouped via Euclidean matrix plots on the basis of mRNA and lncRNA transcripts, and an identical result was observed on the basis of miRNA. This is in accordance with the result of the porcine skeletal and cardiac muscle aging described in a previous study by our group (Chen et al., 2018). These results suggest that gene expression patterns were age-dependent in liver, skeletal, and cardiac muscles. PCA showed a higher degree of variance between young livers compared to old livers based on mRNA and lncRNA transcripts. However, the PCA based on miRNA transcripts showed the opposite result i.e., a higher degree of variance between old livers compared to young livers. A previous study showed that approximately half of the total gene expression changes observed from newborns to age of 40 occurred within the first year of life in the aging process of human brain (Somel et al., 2009). Moreover, it has been reported that the miRNA expression in the frontal cortex appeared to be less affected by aging (Persengiev et al., 2011). occur earlier than those of miRNA, and aging affects the expression of miRNA relatively less in 355 the liver. 
Previous research has shown that age-related genes were involved in immune response,

357 metabolic processes, cell activation, and RNA modification during liver aging in mice (White et

al., 2015), and similar results were found in this study. Here, many age-related genes were

identified during porcine liver aging. GO analysis showed that the up-regulated genes were viruses with age, and the risks for various liver diseases also increased with age (Kim et al., 2015). However, down-regulated genes were mainly involved in metabolism, which may be related to the steady decline of the metabolic ability of the liver (the largest metabolic organ) in response to age. Here, the ELOVL2 gene was found to be significantly down-regulated during porcine liver aging, and it was significantly enriched for the lipid metabolic process. More importantly, previous studies have found that the DNA methylation of the ELOVL2 gene correlated strongly with age in human liver (Bacalini et al., 2018; Bysani et al., 2016). In addition, the ELOVL2 gene also has been found to undergo age-associated hypermethylation on the $\mathrm{CpG}$ island in other tissues (Bacalini et al., 2018), and displays the widest methylation range in response to age in whole blood and white blood cells (Bysani et al., 2016). These results suggest that the reason why the ELOVL2 gene was down-regulated may be closely related to the declining metabolic ability of the liver with age. Furthermore, the hypermethylation of the $\mathrm{CpG}$ island of the ELOVL2 gene may contribute to regulating the ELVOL2 expression in liver aging. Therefore, the ELOVL2 gene may be an important biomarker during liver aging in humans and big mammals. Moreover, the results of this study suggest that several aging-related gene changes may be related to a decline or alteration of the overall function of liver organs. This is a reasonable suggestion based on the obtained results. However, no direct evidence exists that there is a direct relationship between both; therefore, this need further extensive functional 
379

380

381

382

383

384

385

386

387

validations.

Moreover, the nuclear receptor subfamily 1 group H member 3 (NR1H3, ENSSSCG00000013241), encoding LXR- $\alpha$, has been reported to be highly expressed in four samples, and it has been reported that the LXR- $\alpha$ activity and its expression could be repressed by $m i R-206$ in hepatic cells (Vinod et al., 2014). More importantly, a previous study has shown that LXRs as lipid-dependent regulators of inflammatory gene expression may serve to link the lipid metabolism and immune functions in macrophages (Joseph et al., 2003). Interestingly, miR206 was also identified to be up-regulated during porcine liver aging, and several up-regulated genes were also enriched for the immune response in this study. Consequently, this indicates a contribution why the $N R 1 H 3$ was not increased like the $N R 1 H 2$ during porcine liver aging. In addition, the gene $N R I H 2$ was identified to be up-regulated in the liver with increasing age, and the NR1H2 encodes LXR- $\beta$, which is universally expressed (Kalaany \& Mangelsdorf, 2006). A deficient glucose-dependent insulin secretion and an enhanced lipid accumulation have been reported in LXR- $\beta$ knockout mice (Gerin et al., 2005). Furthermore, the variation within the NR1H2 gene may facilitate the development of type 2 diabetes (Ketterer et al., 2011). It has been reported that pigs have a genomic structure, as well as physiological and biochemical features that are similar to those of humans (Welsh et al., 2009); however, pigs cannot develop type 2 diabetes. The obtained results indicate that the increased NR1H2 expression in the aging liver may be one of the key effects involved in the resistance to the development of type 2 diabetes.

In this study, the research animal is the Yanan pig, which has been continuously raised from long ago in the hilly regions of the Sichuan Province. During the last two decades, the production of The Yanan pig has been reduced due to its poor carcass composition and growth performance compared to the white pigs and thus it is believed that the Yanan pig is an endangered pig breed 
402 (Jiang et al., 2012). For this reason, since 2014, the Yanan pig has been included in the National

403 Program for Farm Animal Resources (Yan et al., 2018). Until now, only approximately 100

404 Yanan pigs have been protected in the conservation farm. Here, 180 days and 8 years were

405 selected as the two time points to investigate liver aging, and the investigated sows were healthy

406 and normally growing pigs. The two 180-day-old sows had not previously given birth, while the

407 two 8-year-old sows were in the depleted stage of production performance after many years of

408 giving birth and were eliminated; this enabled the inclusion of two 8-years sows in this research.

409 Although this research offers a meaningful reference for the study of the liver aging process

410 between 14 and 50 years of age in humans, the aging process of the porcine liver needs to be

411 studied at a much older age as it has been shown that the aging rate of the human liver

412 accelerates above the age of 50 (Kudryavtsev et al., 1993). However, 8-years old sows were the

413 oldest available pigs because older sows have been eliminated before due to their depleted

414 production performance. In addition, the Yanan pig is a very treasured pig breed and their

415 population size is very small, which further limited the selection of a higher number of samples

416 in each group for the study of the senescence of pig liver, especially for 8-year-old sows.

417

418

419

420

421

422

423

424

\section{Conclusions}

Aging is a key factor for the development of a large number of diseases, and it can significantly influence the most important metabolic organ the 'liver'. Here, RNA-seq was conducted to determine the expression profiles of mRNA, lncRNAs, miRNAs, and circRNAs. Gene function enrichment analysis showed that up-regulated and down-regulated genes were mainly involved in immune response and metabolism, respectively. A certain number of lncRNAs and their target genes were both observed to be differentially expressed during liver aging. circRNA-miRNA co-expression networks were constructed during liver aging. The

Peer) reviewing PDF | (2018:11:32870:1:2:NEW 4 Apr 2019) 
425

426

427

428

429

430

431

432

433

434

435

436

437

438

439

440

441

442

443

444

445

446

447

448

449

450

451

452

453

454

455

456

457

458

459

460

461

462

463

464

465

466

467 obtained findings contribute to the understanding of the transcriptional basis of liver aging and also provide further references for the understanding of human liver aging at the molecular level.

8

9

0

.

2

\section{References}

Adighibe O, Arepalli S, Duckworth J, Hardy J, and Vrièze WD. 2006. Genetic variability at the LXR gene ( NR1H2 ) may contribute to the risk of Alzheimer's disease. Neurobiology Of Aging 27:1431-1434 DOI 10.1016/j.neurobiolaging.2005.08.010.

Amarapurkar D, Kamani P, Patel N, Gupte P, Kumar P, Agal S, Baijal R, Lala S, Chaudhary D, and Deshpande A. 2007. Prevalence of non-alcoholic fatty liver disease: population based study. Annals of Hepatology 6:161-163 DOI 10.1152/ajpgi.00341.2006.

Bacalini MG, Franceschi C, Gentilini D, Ravaioli F, Zhou X, Remondini D, Pirazzini C, Giuliani C, Marasco E, and Gensous N. 2018. Molecular Aging of Human Liver: An Epigenetic/Transcriptomic Signature. Journals of Gerontology 74:1-8 DOI 10.1093/gerona/gly048

Bertolotti M, Lonardo A, Mussi C, Baldelli E, Pellegrini E, Ballestri S, Romagnoli D, and Loria P. 2014. Nonalcoholic fatty liver disease and aging: Epidemiology to management. World Journal Of Gastroenterology 20:14185-14204 DOI 10.3748/wjg.v20.i39.14185.

Betel D, Wilson M, Gabow A, Marks DS, and Sander C. 2008. The microRNA. org resource: targets and expression. Nucleic Acids Research 36:D149-D153 DOI 10.1093/nar/gkm995.

Bysani M, Perfilyev A, de Mello VD, Rönn T, Nilsson E, Pihlajamäki J, and Ling C. 2016. Epigenetic alterations in blood mirror age-associated DNA methylation and gene expression changes in human liver. Epigenomics 9:105-122 DOI 10.2217/epi-2016-0087.

Capri M, Olivieri F, Lanzarini C, Remondini D, Borelli V, Lazzarini R, Graciotti L, Albertini MC, Bellavista E, and Santoro A. 2017. Identification of miR-31-5p, miR-141-3p, miR-200c-3p, and GLT1 as human liver aging markers sensitive to donor-recipient age-mismatch in transplants. Aging Cell 16:262-272 DOI 10.1111/acel.12549.

Chen G, Wang Z, Wang D, Qiu C, Liu M, Chen X, Zhang Q, Yan G, and Cui Q. 2013. LncRNADisease: a database for long-non-coding RNA-associated diseases. Nucleic Acids Research 41:D983-D986 DOI 10.1093/nar/gks1099.

Chen J, Zou Q, Lv D, Wei Y, Raza MA, Chen Y, Li P, Xi X, Xu H, and Wen A. 2018. Comprehensive transcriptional landscape of porcine cardiac and skeletal muscles reveals differences of aging. Oncotarget 9:1524-1541 DOI 10.18632/oncotarget.23290.

Chen LL, and Yang L. 2015. Regulation of circRNA biogenesis. RNA Biology 12:381-388 DOI 10.1080/15476286.2015.1020271.

Dou C, Cao Z, Yang B, Ding N, Hou T, Luo F, Kang F, Li J, Yang X, and Jiang H. 2016. Changing expression profiles of lncRNAs, mRNAs, circRNAs and miRNAs during osteoclastogenesis. Scientific Reports 6:21499 DOI 10.1038/srep21499.

Eulalio A, Huntzinger E, and Izaurralde E. 2008. Getting to the Root of miRNA-Mediated Gene Silencing. Cell

Peer) reviewing PDF | (2018:11:32870:1:2:NEW 4 Apr 2019) 
132:9-14 DOI 10.1016/j.cell.2007.12.024.

Feng X, Wang Z, Fillmore R, and Xi Y. 2014. MiR-200, a new star miRNA in human cancer. Cancer Letters 344:166-173 DOI 10.1016/j.canlet.2013.11.004.

Friedländer MR, Mackowiak SD, Li N, Chen W, and Rajewsky N. 2011. miRDeep2 accurately identifies known and hundreds of novel microRNA genes in seven animal clades. Nucleic Acids Research 40:37-52 DOI 10.1093/nar/gkr688.

Gerin I, Dolinsky VW, Shackman JG, Kennedy RT, Chiang SH, Burant CF, Steffensen KR, Gustafsson J, and Macdougald OA. 2005. LXR $\beta$ Is Required for Adipocyte Growth, Glucose Homeostasis, and $\beta$ Cell Function. Journal Of Biological Chemistry 280:23024-23031 DOI 10.1074/jbc.M412564200.

Hansen KR, Knowlton NS, Thyer AC, Charleston JS, Soules MR, and Klein NA. 2008. A new model of reproductive aging: the decline in ovarian non-growing follicle number from birth to menopause. Human Reproduction 23:699-708 DOI 10.1093/humrep/dem408.

Huse SM, Gruppuso PA, Boekelheide K, and Sanders JA. 2015. Patterns of gene expression and DNA methylation in human fetal and adult liver. Bmc Genomics 16:981 DOI 10.1186/s12864-0152066-3.

Jiang YZ, Zhu L, Li FQ, and Li XW. 2012. Carcass composition and meat quality of indigenous Yanan pigs of China. Genetics \& Molecular Research 11:166-173 DOI 10.4238/2012.January.27.3

Jones GF, Rothschild MF, and Ruvinsky A. 1998. Genetic aspects of domestication, common breeds and their origin. Oxon: CAB International.

Joseph SB, Castrillo A, Laffitte BA, Mangelsdorf DJ, and Tontonoz P. 2003. Reciprocal regulation of inflammation and lipid metabolism by liver X receptors. Nature Medicine 9:213-219 DOI 10.1038/nm820.

Kalaany NY, and Mangelsdorf DJ. 2006. LXRS and FXR: the yin and yang of cholesterol and fat metabolism. Annual Review Of Physiology 68:159-191 DOI 10.1146/annurev.physiol.68.033104.1 52158.

Ketterer C, Müssig K, Machicao F, Stefan N, Fritsche A, Häring H-U, and Staiger H. 2011. Genetic variation within the NR1H2 gene encoding liver $X$ receptor $\beta$ associates with insulin secretion in subjects at increased risk for type 2 diabetes. Journal of Molecular Medicine 89:75-81 DOI 10.1007/s00109-010-0687-1.

Kim D, Pertea G, Trapnell C, Pimentel H, Kelley R, and Salzberg SL. 2013. TopHat2: accurate alignment of transcriptomes in the presence of insertions, deletions and gene fusions. Genome biology 14:R36 DOI 10.1186/gb-2013-14-4-r36.

Kim H, Kisseleva T, and Brenner DA. 2015. Aging and liver disease. Current Opinion In Gastroenterology 31:184-191 DOI 10.1097/MOG.0000000000000176.

Kitani K. 1994. Aging and the liver: fucntional aspects. Archives of Gerontology \& Geriatrics 19:145-158 DOI 10.1016/0167-4943(94)90036-1.

Kong L, Zhang Y, Ye Z-Q, Liu X-Q, Zhao S-Q, Wei L, and Gao G. 2007. CPC: assess the protein-coding potential of transcripts using sequence features and support vector machine. Nucleic Acids Research 35:W345W349 DOI 10.1093/nar/gkm391.

Kudryavtsev B, Kudryavtseva M, Sakuta G, and Stein G. 1993. Human hepatocyte polyploidization kinetics in the course of life cycle. Virchows Archiv B 64:387-393 DOI 10.1007/BF02915139.

Kwekel JC, Desai VG, Moland CL, Branham WS, and Fuscoe JC. 2010. Age and sex dependent changes in liver gene expression during the life cycle of the rat. Bmc Genomics 11:1-15 DOI 10.1186/1471-2164-11-675.

Li J, Yang J, Zhou P, Le Y, Zhou C, Wang S, Xu D, Lin HK, and Gong Z. 2015. Circular RNAs in cancer: novel insights into origins, properties, functions and implications. American Journal of Cancer Research 5:472-480 DOI,

López-Otín C, Blasco MA, Partridge L, Serrano M, and Kroemer G. 2013. The hallmarks of aging. Cell 153:1194-1217 DOI 10.1016/j.cell.2013.05.039.

Lukiw W. 2013. Circular RNA (circRNA) in Alzheimer's disease (AD). Frontiers in genetics 4:307 DOI 10.3389/fgene.2013.00307.

Lunney JK. 2007. Advances in swine biomedical model genomics. Int J Biol Sci 3:179-184 DOI 10.7150/ijbs.3.179.

Manjula V, Patankar JV, Vinay S, Saša F, Graier WF, Dagmar K, and Kostner GM. 2016. MiR-206 is expressed in pancreatic islets and regulates glucokinase activity. American Journal of Physiology Endocrinology \& Metabolism 311:E175-E185 DOI 10.1152/ajpendo.00510.2015.

Mercer TR, Dinger ME, and Mattick JS. 2009. Long non-coding RNAs: insights into functions. Nature Reviews Genetics 10:155-159 DOI 10.1038/nrg2521.

Mouzat K, Mercier E, Polge A, Evrard A, Baron S, Balducchi JP, Brouillet JP, Lumbroso S, and Gris JC. 
2011. A common polymorphism in NR1H2 (LXRbeta) is associated with preeclampsia. Bmc Medical Genetics 12:145-145 DOI 10.1186/1471-2350-12-145.

Patro R, Duggal G, and Kingsford C. 2015. Salmon: accurate, versatile and ultrafast quantification from RNA-seq data using lightweight-alignment. bioRxiv 10.1101/021592:021592 DOI 10.1101/021592.

Pauter AM, Olsson P, Asadi A, Herslöf B, Csikasz RI, Zadravec D, and Jacobsson A. 2014. Elovl2 ablation demonstrates that systemic DHA is endogenously produced and is essential for lipid homeostasis in mice. Journal Of Lipid Research 55:718-728 DOI 10.1194/jlr.M046151.

Persengiev S, Kondova I, Otting N, Koeppen AH, and Bontrop RE. 2011. Genome-wide analysis of miRNA expression reveals a potential role for miR-144 in brain aging and spinocerebellar ataxia pathogenesis. Neurobiology Of Aging 32:2316. e2317-2316. e2327.

Pertea M, Pertea GM, Antonescu CM, Chang T-C, Mendell JT, and Salzberg SL. 2015. StringTie enables improved reconstruction of a transcriptome from RNA-seq reads. Nature Biotechnology 33:290-295 DOI $10.1038 /$ nbt.3122.

Punta M, Coggill P, Eberhardt R, Mistry J, Tate J, Boursnell C, Pang N, Forslund K, Ceric G, and Clements J. 2012. The Pfam protein families database. Nucleic Acids Research 40:D290-301 DOI 10.1093/nar/gkp985.

Regev A, and Schiff ER. 2001. Liver disease in the elderly. Gastroenterology Clinics Of North America 30:547563 DOI 10.1016/S0889-8553(05)70195-3.

Robinson MD, McCarthy DJ, and Smyth GK. 2010. edgeR: a Bioconductor package for differential expression analysis of digital gene expression data. Bioinformatics 26:139-140 DOI 10.1093/bioinformatics/btp616.

Rodwell GEJ, Sonu R, Zahn JM, Lund J, Wilhelmy J, Wang L, Xiao W, Mindrinos M, Crane E, and Segal E. 2002. A Transcriptional Profile of Aging in the Human Kidney. Plos Biology 12:1566-1573 DOI 10.1371/journal.pbio.0020427.

Sanger HL, Klotz G, Riesner D, Gross HJ, and Kleinschmidt AK. 1976. Viroids are Single-Stranded Covalently Closed Circular RNA Molecules Existing as Highly Base-Paired Rod-Like Structures. Proceedings of the National Academy of Sciences of the United States of America 73:3852-3856 DOI 10.1073/pnas.73.11.3852.

Schmucker DL. 1998. Aging and the liver: an update. Journals of Gerontology 53:B315-320 DOI 10.1093/gerona/53A.5.B315.

Sheedfar F, Biase SD, Koonen D, and Vinciguerra M. 2013. Liver diseases and aging: friends or foes? Aging cell 12:950-954 DOI 10.1111/acel.12128.

Shima M, Hisakazu I, Kiyohito K, Kei N, Mitsuyoshi K, Hirohito Y, Hisaaki M, Joji T, Asahiro M, and Takashi H. 2014. Profile of microRNAs associated with aging in rat liver. International Journal Of Molecular Medicine 34:1065-1072 DOI 10.3892/ijmm.2014.1892.

Slawik M, and Vidal-Puig AJ. 2006. Lipotoxicity, overnutrition and energy metabolism in aging. Ageing research reviews 5:144-164 DOI 10.1016/j.arr.2006.03.004.

Somel M, Franz H, Yan Z, Lorenc A, Guo S, Giger T, Kelso J, Nickel B, Dannemann M, and Bahn S. 2009. Transcriptional Neoteny in the Human Brain. Proceedings of the National Academy of Sciences of the United States of America 106:5743-5748 DOI 10.1073/pnas.09005441 06.

Vinod M, Chennamsetty I, Colin S, Belloy L, Paoli FD, Schaider H, Graier WF, Frank S, Kratky D, and Staels B. 2014. miR-206 controls LXR $\alpha$ expression and promotes LXR-mediated cholesterol efflux in macrophages. BBA - Molecular and Cell Biology of Lipids 1841:827-835 DOI 10.1016/j.bbalip.2014.02.006.

Welsh MJ, Rogers CS, Stoltz DA, Meyerholz DK, and Prather RS. 2009. Development of a porcine model of cystic fibrosis. Transactions of the American Clinical \& Climatological Association 120:149-162.

Wernersson R, Schierup MH, Jørgensen FG, Gorodkin J, Panitz F, Starfeldt H-H, Christensen OF, Mailund T, Hornshøj H, and Klein A. 2005. Pigs in sequence space: a $0.66 \mathrm{X}$ coverage pig genome survey based on shotgun sequencing. BMC genomics 6:70 DOI 10.1186/1471-2164-6-70.

White RR, Milholland B, Macrae SL, Lin M, Zheng D, and Vijg J. 2015. Comprehensive transcriptional landscape of aging mouse liver. Bmc Genomics 16:899 DOI 10.1186/s12864-015-2 061-8.

Woodhouse KW, and James OF. 1990. Hepatic drug metabolism and ageing. British Medical Bulletin 46:22-35 DOI 10.1258/136218005775544589.

Wynne HA, Cope LH, Mutch E, ., Rawlins MD, Woodhouse KW, and James OF. 2010. The effect of age upon liver volume and apparent liver blood flow in healthy man. Hepatology 9:297-301 DOI 10.1002/hep.1840090222.

Yan C, Yingying W, Jianning C, Daojun L, Peining L, Li Z, Guoqing T, Xuewei L, and Yanzhi J. 2018. Growth, carcass characteristics and meat quality of Chinese indigenous Yanan pig crossbred with Duroc and 
Berkshire genotypes. Animal Production Science 10.1071/AN17450 DOI 10.1071/AN17450.

Yun Y, Fan X, Mao M, Song X, Ping W, Yang Z, Jin Y, Yi Y, Chen LL, and Yang W. 2017. Extensive translation of circular RNAs driven by N6-methyladenosine. Cell Research 27:626-641 DOI 10.1038/cr.2017.31.

Zadravec D, Tvrdik P, Guillou H, Haslam R, Kobayashi T, Napier JA, Capecchi MR, and Jacobsson A. 2011. ELOVL2 controls the level of n-6 28:5 and 30:5 fatty acids in testis, a prerequisite for male fertility and sperm maturation in mice. Journal Of Lipid Research 164:S2-S2 DOI 10.1194/jlr.M011346.

Zhang M, Huang N, Yang X, Luo J, Yan S, Xiao F, Chen W, Gao X, Zhao K, and Zhou H. 2018. A novel protein encoded by the circular form of the SHPRH gene suppresses glioma tumorigenesis. Oncogene 37 DOI 10.1038/s41388-017-0019-9.

Zhang X-O, Dong R, Zhang Y, Zhang J-L, Luo Z, Zhang J, Chen L-L, and Yang L. 2016. Diverse alternative back-splicing and alternative splicing landscape of circular RNAs. Genome Research 26:1277-1287 DOI 10.1101/gr.202895.115.

Zhao Y, Alexandrov PN, Jaber V, and Lukiw WJ. 2016. Deficiency in the Ubiquitin Conjugating Enzyme UBE2A in Alzheimer's Disease (AD) is Linked to Deficits in a Natural Circular miRNA-7 Sponge (circRNA; ciRS-7). Genes 7:116 DOI 10.3390/genes7120116.

Zhu J, Chen C, Yang B, Guo Y, Ai H, Ren J, Peng Z, Tu Z, Yang X, and Meng Q. 2015. A systems genetics study of swine illustrates mechanisms underlying human phenotypic traits. BMC genomics 16:88 DOI 10.1186/s12864-015-1240-y.

Zoli M, Magalotti D, Bianchi G, Gueli C, Orlandini C, Grimaldi M, Marchesini G. 1999. Total and functional hepatic blood flow decrease in parallel with ageing. Age \& Ageing 28:29-33 DOI 10.1093/ageing/28.1.29. 
Figure 1

Figure 1: Global mRNA, IncRNA, miRNA, and circRNA expression patterns across liver samples.

(A) mRNA. (B) IncRNA. (C) miRNA. (D) circRNA. (E) Known and novel miRNAs. YL, young liver; $\mathrm{OL}$, old liver. 
A

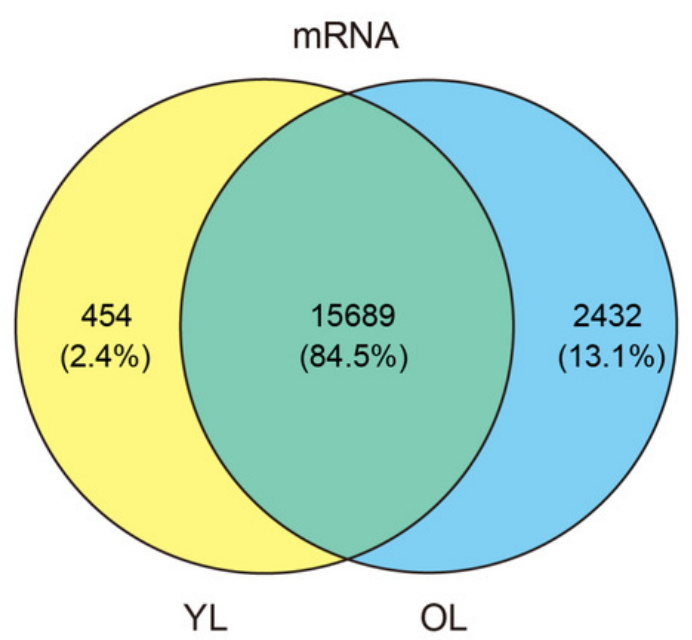

C

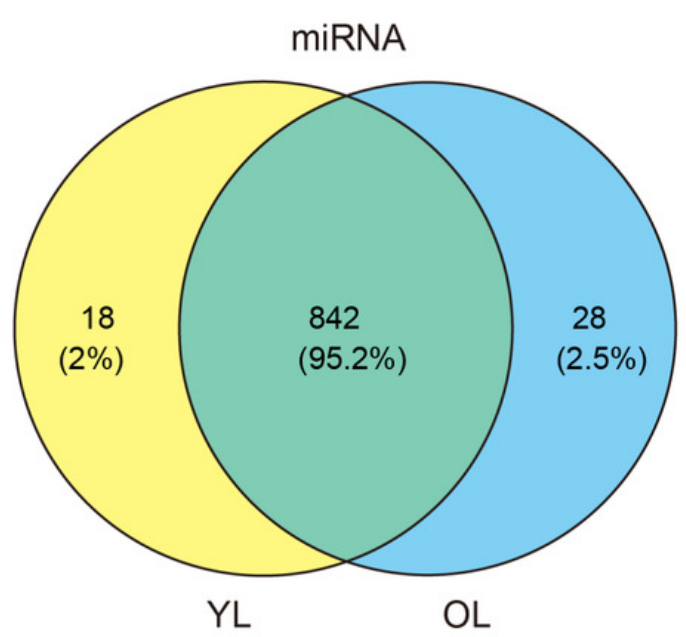

E
B

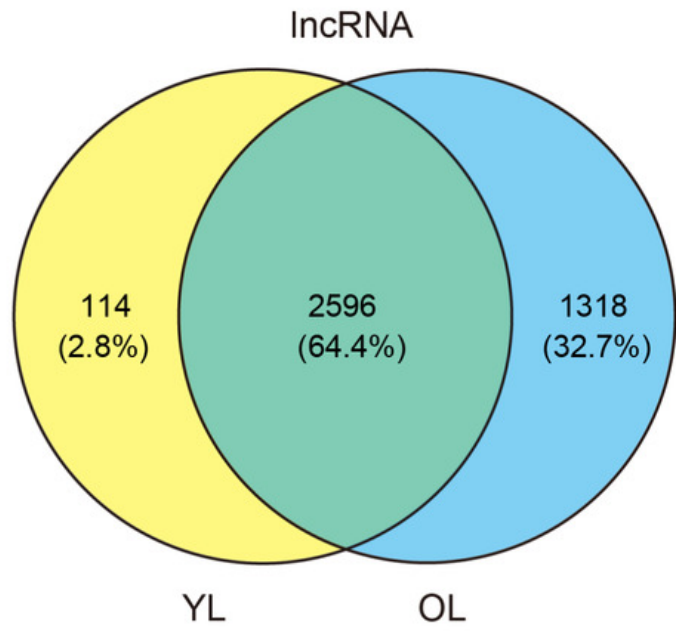

D

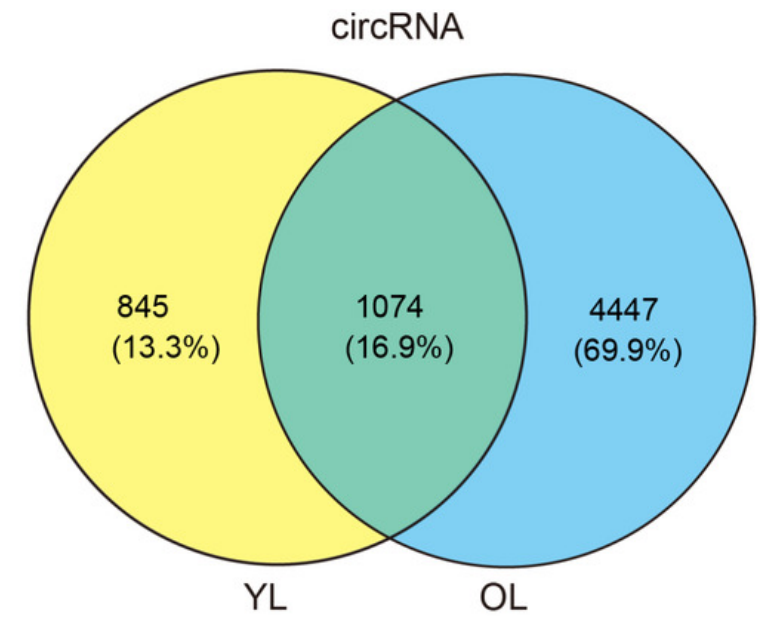

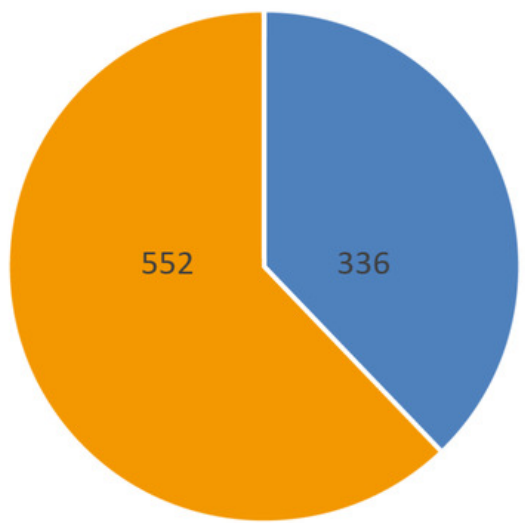

" known miRNA - novel miRNA 


\section{Figure 2}

Figure 2: Variance between old and young individuals.

(A) Pearson's correlation coefficient heat map of mRNA and IncRNA between YL and OL. The identified mRNA and IncRNA expression values (TPM) in every sample were used to perform the Pearson's correlation coefficient analysis, and values closer to 1 were less variable. (B) Principal Component Analysis (PCA) plot based on the normalized expression level (log2 (TPM)) of identified mRNAs and IncRNAs. (C) Pearson's correlation coefficient heat map of miRNAs between $Y L$ and OL. The identified miRNA expression values (TPM) in every sample were used to perform the Pearson's correlation coefficient analysis, and values closer to 1 were less variable. (D) PCA plot based on normalized expression level (log2 (TPM)) of identified miRNAs. 
A

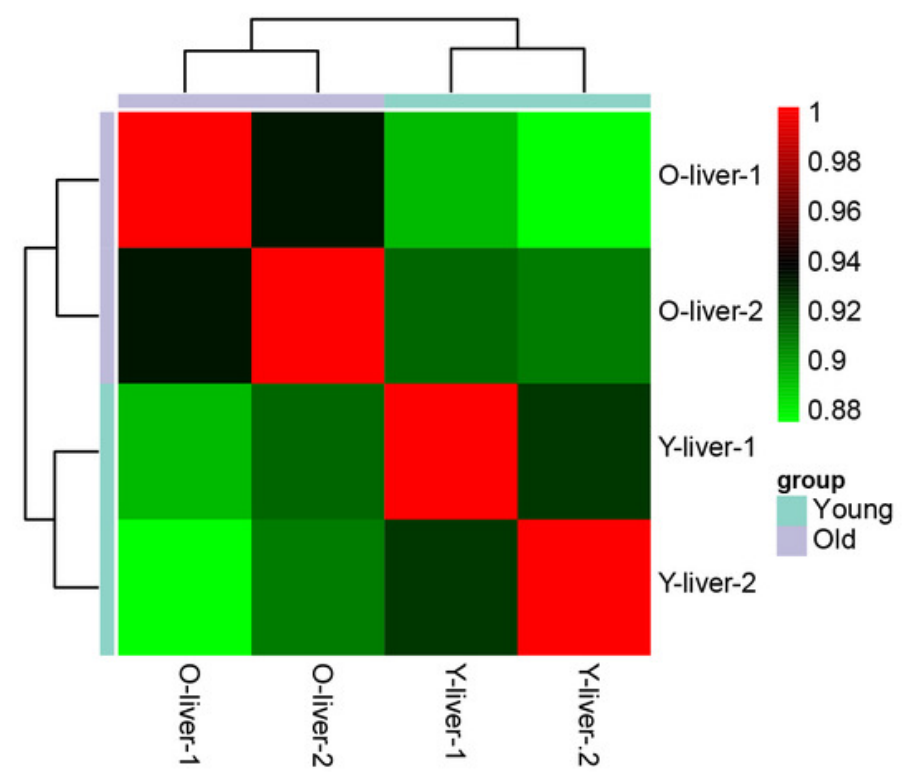

C

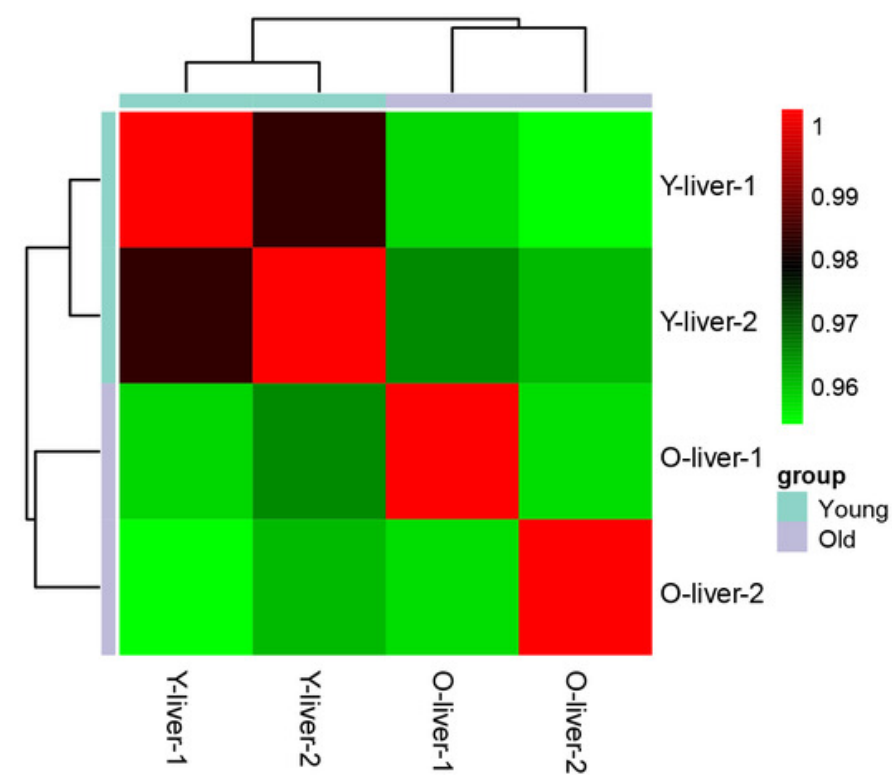

B

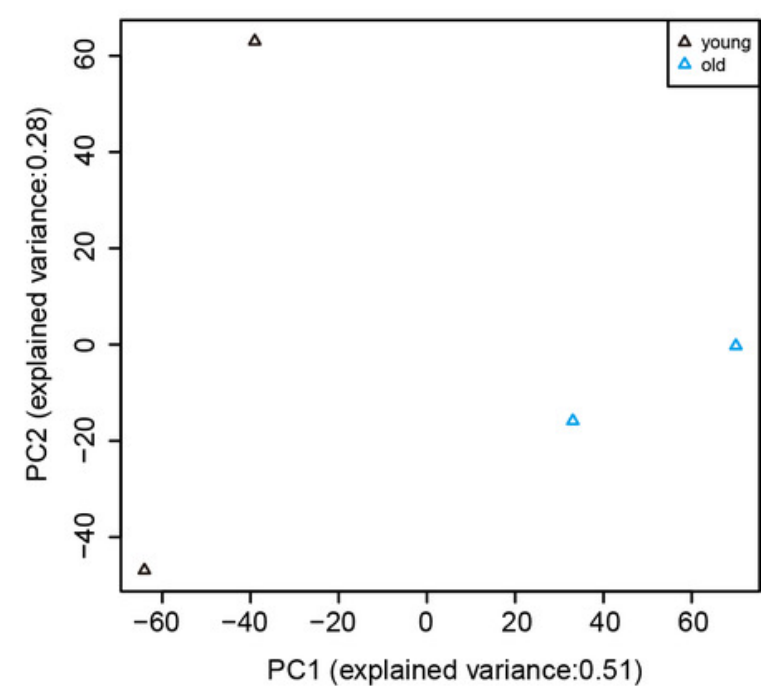

D

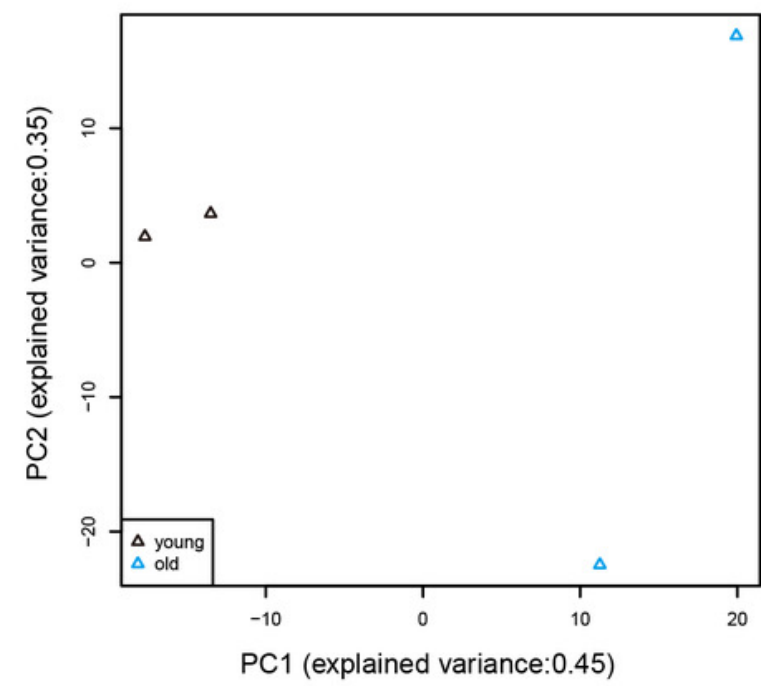




\section{Figure 3}

Figure 3: Differentially expressed mRNAs and IncRNAs during aging.

The $q$-value $<0.05$ and fold change $>2$ were used to identify differentially expressed mRNAs and IncRNAs in OYL. Gene Ontology (GO) enrichment analyses were performed to analyze the functional enrichment of differentially expressed mRNAs and IncRNAs. (A) Differentially expressed mRNAs in OYL. (B) Differentially expressed IncRNAs in OYL. (C) Functional enrichment analysis for up-regulated mRNAs in OYL. (D) Functional enrichment analysis for down-regulated mRNAs in OYL. (E) Function enrichment analysis for the target genes of upregulated IncRNAs in OYL. (F) Function enrichment analysis for the target genes of downregulated IncRNAs in OYL. Only the most enriched $(p<0.05)$ and meaningful GO terms are presented here. OYL, old liver vs. young liver; red dots and blue dots represent up-regulated and down-regulated mRNAs during aging, respectively. FDR, false discovery rate; FC, fold change. BP, biological process; CC, cellular component; MF, molecular function. 
A

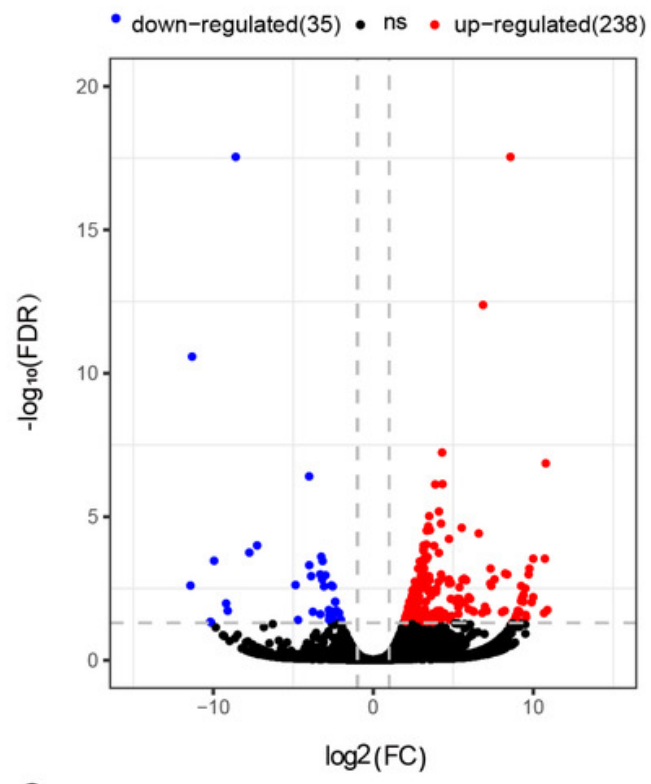

C

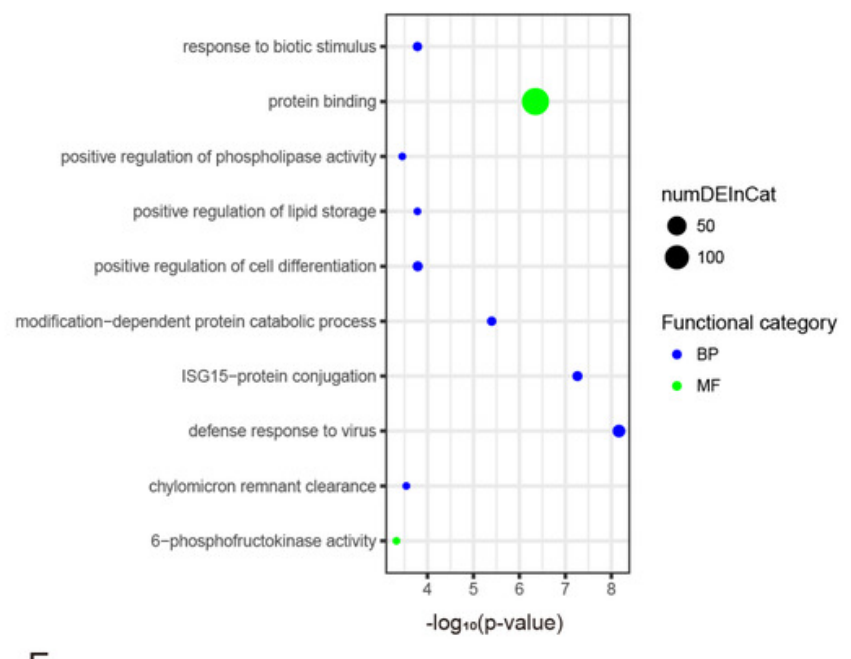

$E$

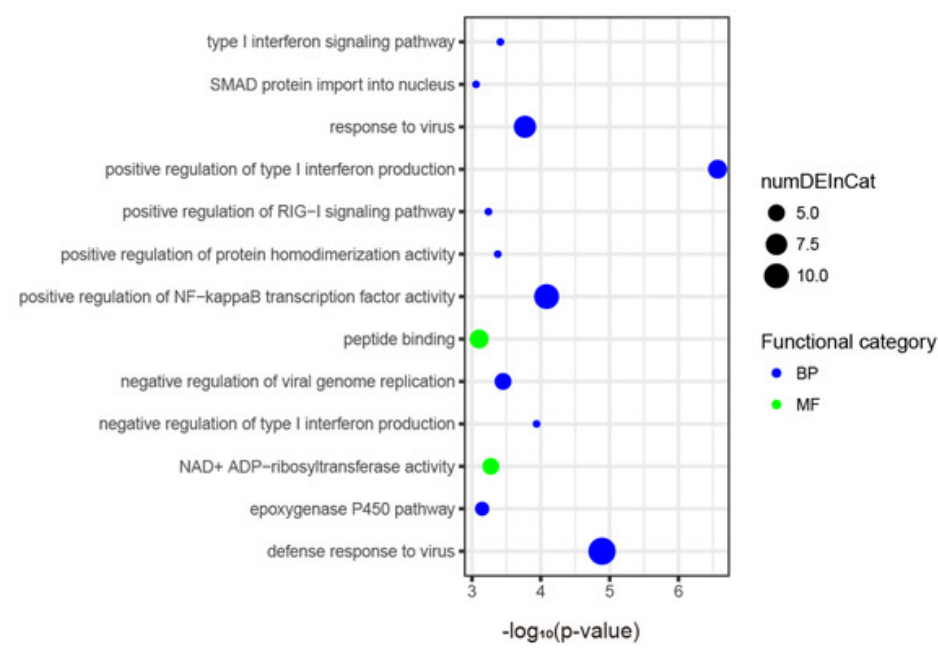

B

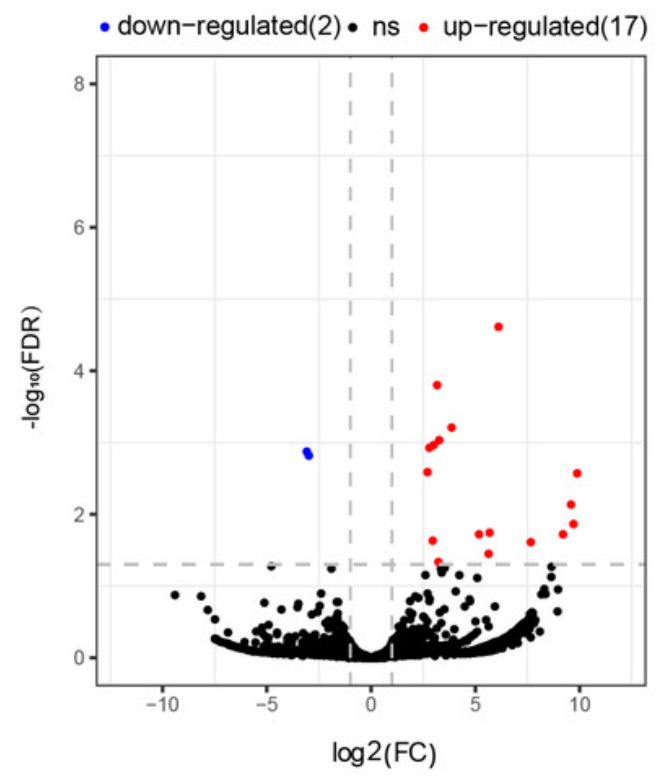

D
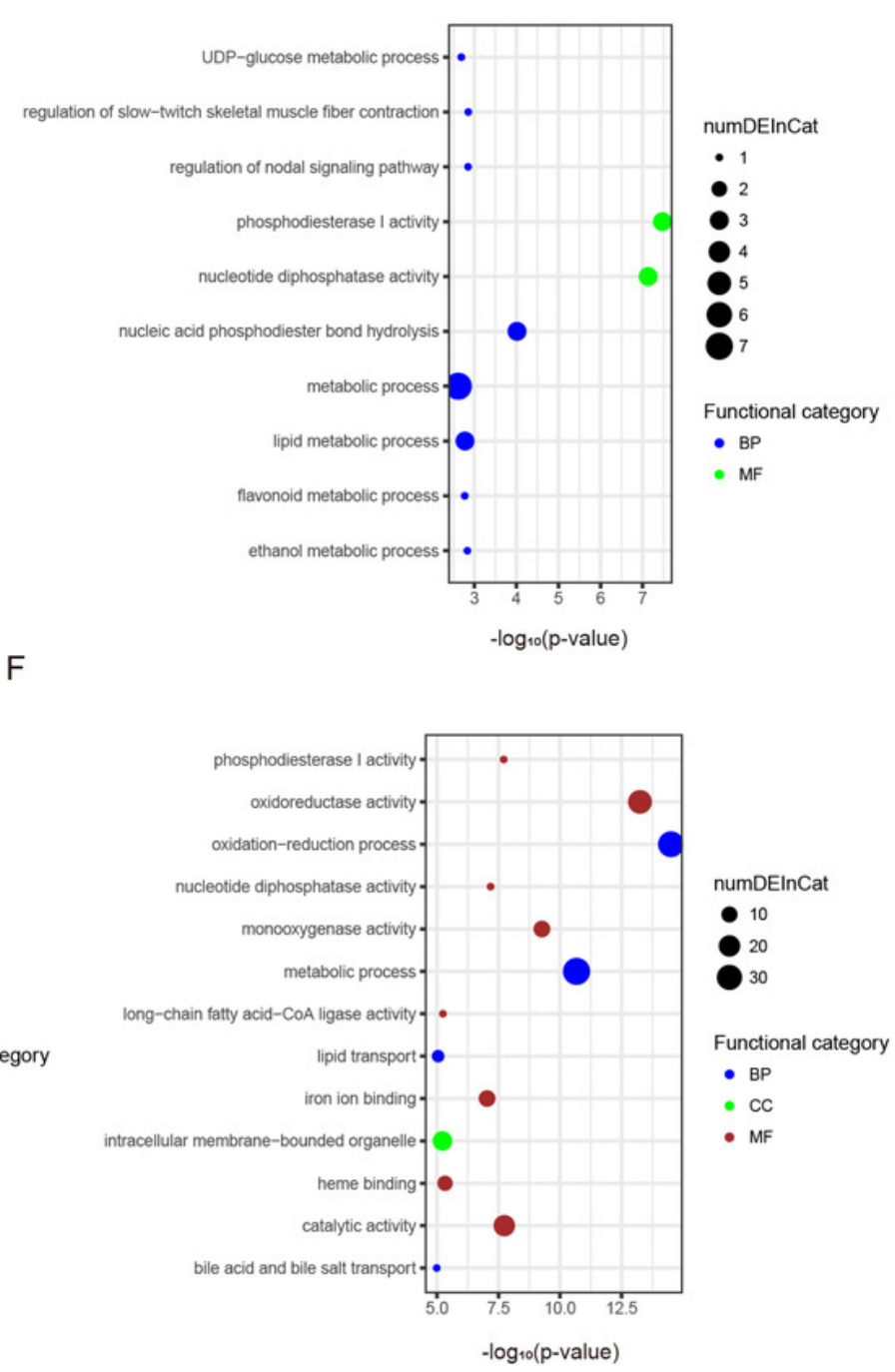


\section{Figure 4}

Figure 4: Construction of the circRNA-miRNA co-expression network in the liver

The Pearson's correlation coefficient analysis was performed between the differentially expressed circRNAs and miRNAs. $r>0.8$ and $p$-value $<0.05$ were considered relevant for the network construction between a circRNA and a miRNA. Red color and blue color represent up and down regulation, respectively. The black solid line represents a positive correlation. 


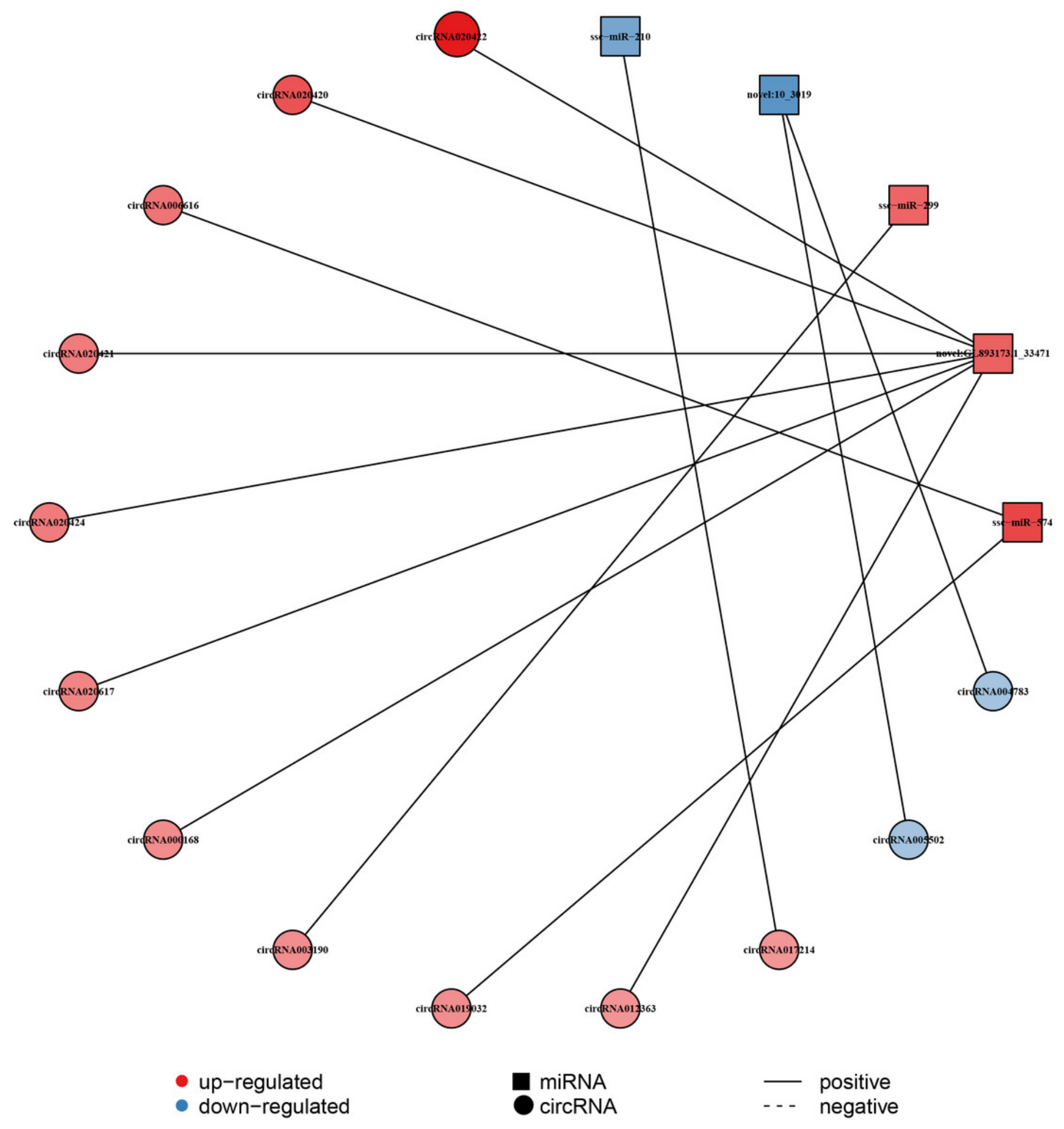




\section{Table $\mathbf{1}$ (on next page)}

Table 1. Gene Ontology annotations of differentially expressed IncRNA and their differentially expressed targets in OYL.

Note: Abbreviations: OYL: old liver vs. young liver; $\uparrow:$ Up-regulated; $\downarrow$ : Down-regulated. 
Table 1. Gene Ontology annotations of differentially expressed IncRNA and their differentially expressed targets in OYL.

\begin{tabular}{|c|c|c|c|c|}
\hline $\operatorname{lncRNA}$ & Targets (corrected p-value) & $\begin{array}{c}\text { Pearson } \\
\text { correlation }\end{array}$ & adjusted $p$-value & Gene Ontoloty annotations \\
\hline \multicolumn{5}{|l|}{ OYL } \\
\hline \multirow[t]{3}{*}{ MSTRG.58269个 } & DMBX1 (ENSSSCG00000003900)^ & 0.955329954 & 5.67E-07 & DNA binding \\
\hline & GUCA1A ( ENSSSCG00000001636)^ & 0.949969984 & $3.66 \mathrm{E}-06$ & $\begin{array}{l}\text { Calcium sensitive guanylate cyclase activator } \\
\text { activity }\end{array}$ \\
\hline & IRG6 ( ENSSSCG00000008648)^ & 0.925610513 & 0.001682339 & Defense response to virus \\
\hline \multirow[t]{3}{*}{ MSTRG.85782个 } & ISG15 (ENSSSCG00000027982) $\uparrow$ & 0.944967314 & $1.69 \mathrm{E}-05$ & Defense response to virus \\
\hline & S1PR3 (ENSSSCG00000009580)^ & 0.926961232 & 0.001284965 & Regulation of interleukin-1 beta production \\
\hline & IRG6 (ENSSSCG00000008648)^ & 0.921249274 & 0.003855634 & Negative regulation of viral genome replication \\
\hline MSTRG.177174 & SARDH (ENSSSCG00000005740)^ & 0.949551978 & 4.19E-06 & Oxidation-reduction process \\
\hline \multirow[t]{5}{*}{ MSTRG.205482 } & CYP1A1 (ENSSSCG00000001906) $\downarrow$ & 0.993093224 & $1.40 \mathrm{E}-23$ & Hydrogen peroxide biosynthetic process \\
\hline & 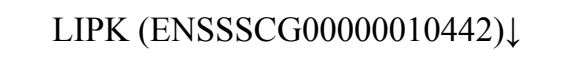 & 0.990169559 & $5.79 \mathrm{E}-20$ & Lipid metabolic process \\
\hline & SULT1B1 (ENSSSCG00000027194) $\downarrow$ & 0.985193868 & $4.78 \mathrm{E}-16$ & Flavonoid metabolic process \\
\hline & TLR4 (ENSSSCG00000024231) $\downarrow$ & 0.949426725 & $4.36 \mathrm{E}-06$ & Lipopolysaccharide receptor activity \\
\hline & ENPP3 (ENSSSCG00000004194) $\downarrow$ & 0.94060909 & $5.60 \mathrm{E}-05$ & Nucleotide diphosphatase activity \\
\hline
\end{tabular}

Note: Abbreviations: OYL: old liver vs. young liver; $\uparrow:$ Up-regulated; $\downarrow$ : Down-regulated. 